\title{
El arte terapia en el desarrollo motor en los niños con síndrome de down de 5 años de edad en la Fundación Corazón de María de Pelileo.
}

The art therapy in motor development in children with Down syndrome from 5 years of age in the Foundation Heart of Mary of Pelileo.

Troya Ortiz Elsa Verónica. , Aguiar Gaibor Viviana Carolina. , Peñafiel Gaibor Víctor. , Miño Acurio Jeanette Alexandra. Ana Mercedes Avila Chillo. \& Rodrigo Daniel Argotti Zumbana.

Recibido: 11-03-2017 / Revisado: 10-05-2017 Aceptado: 05-06-2018/ Publicado: 01-07-2018

\section{Abstract.}

DOI: $\underline{\text { https://doi.org/10.33262/cienciadigital.v2i3.173 }}$

This research project is I developed on the theme "Art Therapy motor development in children with Down syndrome 5 years old at the Heart Foundation María of Pelileo" had as its overall objective to establish how it influences the art Therapy in motor development in children with Down syndrome 5 years of age in the Heart of Mary Pelileo Foundation, and as specific objectives: to assess the level of motor development in children with Down syndrome to identify what techniques Art Therapy used to develop skills and abilities in children 5 years old with Down syndrome, associating the influence of art therapy with the motor development of children with Down syndrome. We worked with a population of 10 children 5 years of age, this research is descriptive with qualitative - quantitative approach. Information was collected by performing a pre-test and post-test by Nelson Ortiz Scale applied to children. Resulting in the Art Therapy if it influences and is helpful in developing gross motor and fine motor development of the child as the statistical data indicates that if there is a significant difference after we applied the art therapy for children 5 years with Down syndrome.

Keywords: Art Therapy, Gross Motor, Motor Fine, Children, Down's Syndrome.

\section{Resumen.}

El presente Proyecto de Investigación se desarrolla sobre el tema "El Arte Terapia en el Desarrollo Motor en los niños con Síndrome de Down de 5 años de edad en la 
Fundación Corazón de María de Pelileo", tuvo como objetivo general establecer cómo influye el Arte Terapia en el Desarrollo Motor en los niños con Síndrome de Down de 5 años de edad en la Fundación Corazón de María de Pelileo, y como objetivos específicos: evaluar el nivel de desarrollo motor en los niños con Síndrome de Down, identificar qué técnicas se utiliza en el Arte Terapia para el desarrollo de habilidades y destrezas en los niños de 5 años con Síndrome de Down, asociar la influencia del Arte Terapia con el desarrollo motor del niño con Síndrome de Down. Se trabajó con una población de 10 niños de 5 años de edad, esta investigación es de tipo descriptiva con un enfoque cuali - cuantitativo. Se recogió la información realizando un pre-test y un post-test a través de la Escala de Nelson Ortiz aplicados a los niños. Obteniendo como resultado que el Arte Terapia si influye y es de gran ayuda en el desarrollo motor grueso y desarrollo motor fino del niño ya que los datos estadísticos nos indica que si existe una diferencia significativa después de que se les aplicó el Arte Terapia a los niños de 5 años con Síndrome de Down.

Palabras Claves: Arte Terapia, Motor Grueso, Motor Fino, Niños, Síndrome de Down

\section{Introducción .}

Desde la antigüedad se hablado del arte terapia como una estrategia para tratar enfermedades de forma terapéutica, siendo más notorio a partir de la segunda guerra mundial debido a la experiencia que tuvieron cuando muchos de los convalecientes que llegaron a los hospitales se comunicaban a través de dibujos.

En los años cuarenta y cincuenta del siglo XX, en Estados Unidos Margaret Naumburg y Edith Kramer pusieron las primeras piedras del arte terapia como una disciplina académica. Naumburg (1977) habla de los primeros programas de formación en arte terapia que se organizaban en ese momento en una facultad de medicina, estos cursos comprendían una preparación en psicología avanzada, psiquiatría clínica, y estudios sobre trabajos en artes plásticas y visuales actualmente existen muchos programas para que las personas puedan tener una formación en arte terapia en distintas universidades, todos estos programas están controlados por la Asociación Americana de Arte Terapia. (1)

La Asociación Americana de Arte Terapia (AATA) define a esta ciencia así: "El arteterapia es una profesión establecida en la salud mental que ocupa los procesos creativos de la realización de arte para mejorar y explorar el bienestar físico, mental y emocional de individuos". Jean-Pierre Klein (2008) define en su libro al arte terapia como: "Una búsqueda de sí que no está ahí por anticipado, no está sino como una posibilidad".

El arte terapia es un tratamiento profundo, que se realiza de forma individualizada, en el cual el paciente acude a varias sesiones en las que el terapeuta con ejercicios interioriza con el paciente y hace que trabaje y se ponga en contacto con su imaginación y creatividad. 
Guilford (1950) "La personalidad creativa se define según la combinación de rasgos característicos de las personas creativas. El arte terapia aparece de una conducta creativa en la que incluye actividades como la organización, la planificación, la invención, la composición y la elaboración”.

José Barragán menciona "El arte como una forma de terapia nos ofrece la oportunidad para enlazar nuestra experiencia personal o intrapersonal con experiencias ajenas. Esto constituye la posibilidad de poder interpretar lo que se siente entre las vivencias que a veces nos parecen incomunicables, esto mismo lo puede sentir una persona en cualquier contexto de intervención social, terapéutico o educativo, tanto los arteterapeutas como los pacientes.

Por lo tanto hay que concebir el arte como historias abiertas y no como un texto cifrado que podemos decodificar sino como un condensado de experiencias que pueden generar infinitas interpretaciones y nos permita comprendernos mejor a nosotros mismos y entre nosotros.

Según los textos citados por los autores, he llegado a la conclusión, que el arte terapia es un medio por el cual todas las personas sin importar su edad ni su condición física o psíquica pueden expresar sus habilidades, destrezas, imaginación y creatividad, lo cual va a ser de gran ayuda no solo en el desenvolvimiento de sus áreas de desarrollo sino también en su personalidad y autoestima, ya que la persona se sentirá mejor consigo mismo y con las demás personas que se encuentran en su entorno.

La motricidad fina comprende todas aquellas actividades del niño que necesitan de una precisión y un elevado nivel de coordinación, es decir una actividad armónica de partes que cooperan en una función, sobre todo de los grupos musculares bajo la dirección cerebral.

Implica un nivel elevado de maduración y un aprendizaje largo para la adquisición plena de cada uno de sus aspectos, ya que hay diferentes niveles de adquisición y dificultades.

Y es el arte terapia como una ciencia y como un método novedoso, es de gran ayuda para personas con discapacidades sobre todo para niños que posean alguna discapacidad, porque así podrán poner en práctica sus habilidades desde muy temprana edad de una manera diferente y divertida, a través de la cual se descubrirán y sabrán cuál o cuáles son sus fortalezas en el ámbito del arte, además de mejorar su desarrollo evolutivo y su parte emocional.

El arte terapia es de gran interés no solo para arteterapeutas sino también para profesionales tales como: psicólogos, psiquiatras, y estimuladores tempranos porque a través del arte terapia podemos seguir trabajando con los niños en cada una de sus áreas pero de una manera innovadora, creativa y divertida, por lo que es un método que puede complementar 
a nuestro trabajo y ser de gran aporte en la ayuda que brindamos a cada uno de los niños. (10)

Edith Kramer es quien empieza a trabajar con niños y pre-adolescentes difíciles, con terapia a través del arte, teniendo como meta una rehabilitación y el crecimiento de la personalidad de cada uno de ellos, y es quien empieza los estudios de arte terapia en la universidad de los Estados Unidos.

Desde entonces el arte terapia ha ido evolucionando y dándose a conocer en ciertos países, en los cuales se ha ido trabajando con personas de varias edades y también con personas que padecen discapacidades en las cuales se ha visto una gran evolución. ${ }^{(8)}$

En la actualidad el arte terapia está dándose a conocer por sus múltiples beneficios y virtudes sobre todo en niños con discapacidades especiales con los cuales los arteterapeutas están trabajando y pueden ver como los niños mejoran en su desarrollo y en su personalidad.

Rubin (1999) comenta que en Inglaterra en el año de 1930 y 1950 el arte terapia es una modalidad que conjuga varias técnicas para el desarrollo del niño, aun mas en personas con discapacidades, quienes elaboraron trabajo artístico y debido a esto el arte terapia apareció como una necesidad general para entender asuntos con mayor profundidad. (1)

Múltiples son los beneficios mejora la coordinación motriz y la manera de controlar el cuerpo, brinda la posibilidad de desarrollar su percepción y mediante la experiencia artística se desarrolla la observación visual: sensibilidad al color, a la forma, al espacio, al mundo que nos rodea.

También se desarrollan los otros sentidos, como el táctil, que las ayudas a percibir la materia y modelar sus propias creaciones, también se desarrollan los otros sentidos, como el táctil, que los ayuda a percibir la materia y modelar sus propias creaciones.

El niño es un ser dinámico y creativo, para él jugar es un medio de comunicarse aprender, aunque por mucho tiempo se ha visto el juego solo como un pasa tiempo y no se le ha dado la importancia necesaria, se centra alrededor de su cuerpo y facilita el aumento de las habilidades motrices y físicas, aspectos en los que se divide el desarrollo psicomotor, el cual se refiere a la enseñanza del movimiento con control y eficiencia en el espacio. Las habilidades motrices se refieren a un nivel en el que el niño es capaz de manifestarse con una considerable facilidad y eficiencia en su ambiente. (11)

Gallahue (1987) divide estas habilidades en tres categorías:

Estabilidad: Es la habilidad de mantener el equilibrio en relación con la fuerza de la gravedad, aunque la aplicación natural de la fuerza pueda alterar las partes del cuerpo en una posición inusual.

Locomoción: Cambios en la localización del cuerpo en relación con puntos fijos del suelo, incluye la proyección del cuerpo en el espacio externo. 
Manipulación: Implica dar y recibir fuerza de objetos mediante el uso de manos y/o pies. La adquisición de destrezas psicomotrices se considera como un proceso a través del cual el niño aprende a construir secuencias de movimiento que cuando sea adolescente le servirá para una vida activa.

Comellas y Perpinyá (1984) comentan que el niño inicia la motricidad fina hacia el año y medio cuando sin ningún aprendizaje empieza a emborronar y pone bolitas o cualquier objeto pequeño en un frasco o botella.

\section{Coordinación Viso-manual .}

El hecho de extender la mano para agarrar algo exige la coordinación ojo-mano, ésta coordinación consiste en la cooperación de los ojos y de las manos de tal forma que los primeros dirigen los movimientos de las ultimas. (11)

La motricidad gruesa es un área fundamental ya que esta se encarga del control de los grandes grupos musculares y por ende a los grandes movimientos que se realizan como correr, lanzar, saltar, habilidades que son la base de todas las actividades físicas y también de las posteriores habilidades deportivas. Al ser el pilar de todos los movimientos, la motricidad gruesa debe ser estimulada y controlada en los niños desde pequeños buscando el desarrollo de los músculos de todo el cuerpo, especialmente de los miembros inferiores.

El día de hoy el Síndrome de Down es la causa más frecuente que se conoce de retraso mental, y es importante tratar sobre el lenguaje y la comunicación ya que los mismos son temas claves que limitan el desarrollo personal y social del niño Down.

Ciertamente a través del dibujo el niño expresa su conocimiento sobre el mundo y sobre sí mismo, por lo que la representación de detalles y objetos tiene que ver con la capacidad intelectual en los niños con Síndrome de Down se presenta un retardo del área cognitivo siendo primordial brindar estímulos en edades tempranas, de ahí la importancia de utilizar el arte terapia como estrategia para aumentar las capacidades, habilidades y destrezas que se generan a partir del desarrollo motor de los niños.

Con estas premisas la presente investigación trató un tema trascendental como es el Arte Terapia en el Desarrollo Motor en los niños con Síndrome de Down de 5 años de edad que acuden a la Fundación Corazón de María de Pelileo.

Se pretendió dar una nueva posibilidad de acción terapéutica pensando la relación entre Motricidad y el Arte terapia, la cual se produce a partir de la experiencia sensoriales de los niños de tal forma que comprendan, entiendan el ambiente que lo rodea a través del proceso de creación, considerando las características marcadas que tienen los niños con Síndrome de Down (SD de aquí en adelante) tanto en su crecimiento y desarrollo. 


\section{Metodologìa.}

El estudio fue descriptivo longitudinal de campo ya que se realizó en el lugar de los hechos para establecer e identificar la relación entre la variable independiente y dependiente de tal forma que se pueda verificar si la hipótesis planteada se cumple o no, se trabajó con 10 niños/as de 5 años que pertenecen a la Fundación Corazón de María de Pelileo, de los cuales se consideró criterios de inclusión a los niños y niñas de 5 años de edad con Síndrome de Down inscritos en la Fundación Corazón de María de Pelileo.

Los niños-as cuyos padres hayan otorgado el consentimiento para la participación en el estudio fueron incluidos, y los niños que fueron considerados como criterio de exclusión fueron los niños mayores de 5 años, niños aparentemente normales y niños cuyos familiares no aceptaron la participación en el estudio.

Para el cumplimiento de los objetivos planteados primero se dio a conocer a los padres de familia sobre el tipo de investigación para que firmen un consentimiento informado, posteriormente se aplicó la escala de Nelson Ortiz instrumento aprobado por la Unicef la cual mide el nivel de desarrollo de los niños en varias áreas.

La escala abreviada de desarrollo aplicada consta de nueve partes: una escala que se aplica de 0 a 3 meses, de 3 a 6 meses, de 6 a 9 meses, de 9 a 12 meses, de 12 a 18 meses, de 18 a 24 meses, de 24 a 36 meses, de 36 a 48 meses, de 48 a 60 meses, cada escala consta de ítems del desarrollo acorde a la edad, los cuales se irán evaluando hasta que el niño falle en por lo menos tres ítems consecutivos, ahí se suspende. Se evaluará con los siguientes puntajes frente al ítem evaluado: 1: lo hace y 0: no lo hace, sumamos el número de ítems en total con esto se establece si el niño está en el rango de alerta, medio, medio alto, o alto.

Seguido a esto se planificó actividades utilizando varias técnicas basadas en la Música, Danza, Teatro, Pintura sabiendo que las mismas forman parte del arte terapia, y se las realizó de forma individual o grupal con los niños con Síndrome de Down.

Las sesiones de arte terapia, cumplieron una serie de condiciones prácticas denominadas en el argot de la profesión "setting" (encuadre), éstas condiciones garantizan el desarrollo de un ambiente de seguridad, confianza, y concentración en las sesiones, lo cual permitirá el proceso terapéutico.

Tanto para las sesiones individuales o grupales se realizó al menos una sesión semanal de una hora de duración, pudiendo ampliarse dos sesiones semanales de una hora.

La duración de la terapia duró de acuerdo a las necesidades del niño, existiendo procesos muy breves de 10 o 12 sesiones, y procesos largos de 30 sesiones en adelante, considerando que el aprendizaje, interiorización en los niños con este síndrome lleva el trabajo constante $\mathrm{y}$ repetitivo. 
Para las sesiones se usó salas amplias, equipadas con mesas, sillas y todos los materiales necesarios los cuales estaban ubicados en un armario que debe estar a disposición de los niños, en donde también se ubicarán los objetos artísticos creados en las sesiones de Arteterapia.

Los materiales básicos para las sesiones de Arte terapia son: Lápices de colores, Borradores, Lápices de carbón, Acuarelas, Temperas, Pinceles, Brochas, Recipientes de plástico, Barro, Papel A4 blanco, Cartulinas A3 de colores, Obras de teatro, cuentos, CD de música bailable, relajación.

Aspectos éticos que se consideró:

- Se presentó la metodología, el test y el consentimiento informado para los padres de familia, al personal de la Fundación Corazón de María de Pelileo, los mismos que fueron aprobados

- Los niños y niñas que participaron no fueron sometidos a riesgos por que fue un estudio de observación

- La investigadora realizó el estudio demostrando calidad humana, solvencia ética y eficiencia.

- Se garantizó la confidencialidad de la información y su uso exclusivo para la presente investigación.

- La investigación se desarrolló de acuerdo al diseño establecido en el protocolo.

- Al momento de realizar el trabajo de campo se registró la información en la ficha individual del niño o niña; no se hizo comparaciones con respecto a otros niños y se mantuvo la confiabilidad de la información de cada uno de los niños Información que se proporcionó a los padres de los niños participantes.

- Se informó que se trata de un estudio que busca determinar cómo influye el Arte Terapia en el Desarrollo Motor en niños con Síndrome de Down que asisten a la Fundación Corazón de María de Pelileo.

- Se aclaró que la participación de los niños es voluntaria y que se pueden retirar en el momento que deseen. En el estudio no se presentaron acontecimientos adversos.

\section{Resultados y discusión.}

Una vez terminado el proyecto de investigación hemos llegado a los siguientes resultados según las evaluaciones a los niños con la Escala de Nelson Ortiz: 
Gráfica 1: Área Motriz Gruesa

\begin{tabular}{|l|r|}
\hline \multicolumn{1}{|c|}{ Pre-test } & Niños \\
\hline Alerta & 7 \\
\hline Medio & 2 \\
\hline Medio alto & 1 \\
\hline Alto & 0 \\
\hline
\end{tabular}

Fuente: Pre-Test Escala Nelson Ortiz

Elaborado por: Viviana Aguiar

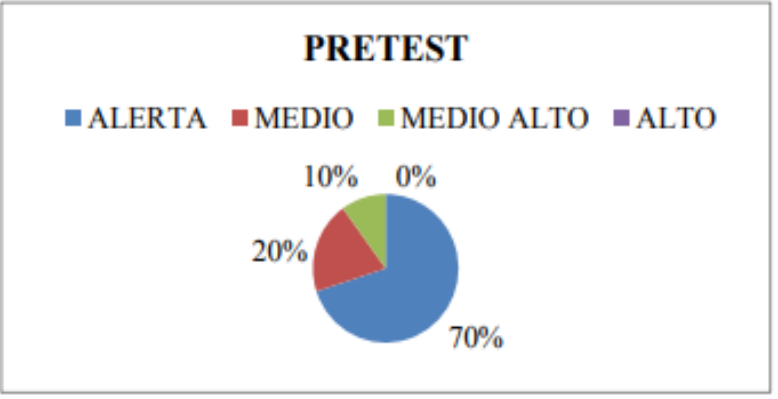

Fuente: Post- Test Escala Nelson Ortiz

Elaborado por: Viviana Aguiar.

. Gráfica 2: Área Motriz Gruesa

\begin{tabular}{|c|c|}
\hline Post-Test & Niños \\
\hline Alerta & 2 \\
\hline Medio & 6 \\
\hline Medio Alto & 2 \\
\hline Alto & 0 \\
\hline
\end{tabular}

\section{POST-TEST}

@ALERTA $\square$ MEDIO $\square$ MEDIO ALTO $\square$ ALTO

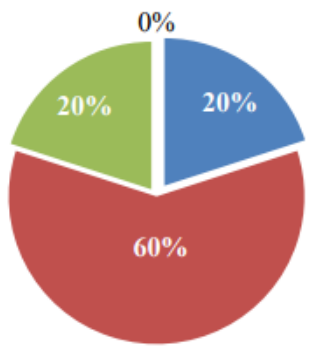


Fuente: Post- Test Escala Nelson Ortiz

Elaborado por: Viviana Aguiar.

Tabla 1: Análisis de los resultados del Área Motriz Gruesa.

\begin{tabular}{ccc}
\hline Niños & Pre-test & Post-test \\
\hline 1 & 26 & 26 \\
2 & 25 & 26 \\
3 & 26 & 29 \\
4 & 25 & 27 \\
5 & 24 & 28 \\
6 & 26 & 27 \\
7 & 25 & 29 \\
8 & 27 & 28 \\
9 & 28 & 30 \\
10 & 30 & 30 \\
\hline
\end{tabular}

Fuente: Escala de Nelson Ortiz.

Discusión: Según los datos obtenidos del análisis con la t-student se pudo observar que en los 10 niños con Síndrome de Down que fueron evaluados con un pre-test y un post-test con la escala de Nelson Ortiz se encuentra que si existe influencia del Arte Terapia en el desarrollo motor grueso de los infantes.

Grafica 3: Área Motriz Fina.

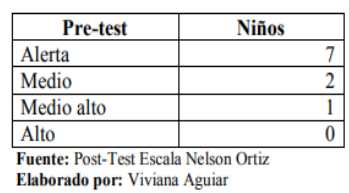

PRE-TEST

-ALERTA $\|$ MEDIO $\square$ MEDIO ALTO $\|$ ALTO

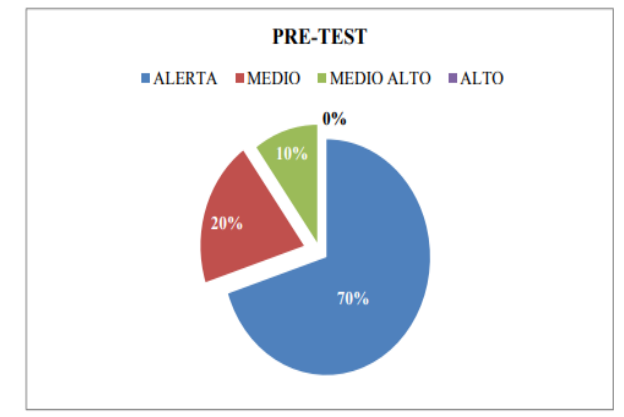


Fuente: Post- Test Escala Nelson Ortiz

Elaborado por: Viviana Aguiar.

Grafica 4: Área Motriz Fina.

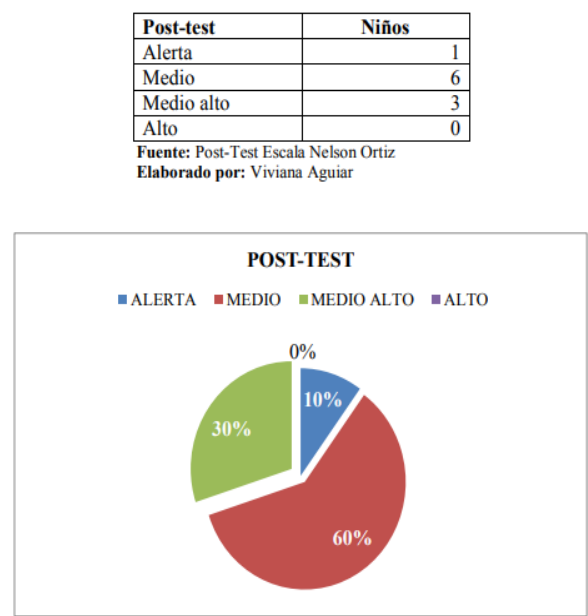

Fuente: Post- Test Escala Nelson Ortiz

Elaborado por: Viviana Aguiar.

Tabla 2: Análisis de los resultados del Área Motriz Fina

\begin{tabular}{ccc}
\hline Niños & Pre-tet & Post-test \\
\hline 1 & 25 & 26 \\
2 & 25 & 29 \\
3 & 26 & 27 \\
4 & 26 & 28 \\
5 & 24 & 27 \\
6 & 26 & 27 \\
7 & 25 & 27 \\
8 & 28 & 30 \\
\hline
\end{tabular}




\begin{tabular}{ccc}
\hline 9 & 29 & 30 \\
10 & 30 & 30 \\
\hline
\end{tabular}

Fuente: Escala de Nelson Ortiz

Discusión: Se aplicó la t-student obteniendo como resultado que el Arte Terapia si influyó en gran parte en el desarrollo motor fino de los 10 niños con Síndrome de Down de 5 años de edad.

Estos resultados son significativos ya que se realizó un estudio investigativo con el objetivo de determinar qué efectos causa el arte terapia en las habilidades de niños con síndrome de Down. Se aplicó la técnica de la encuesta, usando el cuestionario de Habilidades Sociales de Guillen (2008), el cuestionario tuvo preguntas con cuatro alternativas de respuesta.

Al comparar el test antes y después de realizarlo se encontraron diferencias significativas, las cuales atribuyeron al programa de arte terapia como tratamiento, concluyendo que el arte terapia aumenta las habilidades de niños con síndrome de Down. Los resultados que se obtuvieron pueden ser de gran utilidad para psicólogos, psicopedagogos, o para quien esté interesado en usar el arte como terapia. (7)

Si para Guilford (1950) la personalidad creativa se define según la combinación de rasgos característicos de las personas creativas. El arte terapia aparece de una conducta creativa en la que incluye actividades como la organización, la planificación, la invención, la composición y la elaboración, en el estudio se evidencia que con las actividades planteadas se fomenta el desarrollo no solo motor sino de todas las áreas del desarrollo dando un enfoque integral en el niño y del cual su familia también fue participe.

Y considerando varios estudios que han realizado en otros países se comprobó la hipótesis que se planteó en el trabajo; Corroborando además con María Alejandra Marín, la única terapeuta reconocida en la ciudad de Guayaquil, la cual menciona que "El arte terapia es relativamente nuevo y se están abriendo espacios para fomentar el arte como medio de expresión y de cura; en lo personal la aplicó debido a observaciones y competencias sólidas en el área de pintura y dibujo lo que facilitó el proceso de enseñanza, más la colaboración de los directores que aprobaron la iniciativa”.(5)

Por tanto el arte como terapia es un gran método para poder incentivar a las personas a potenciar o fortalecer sus destrezas, sus habilidades, su imaginación y creatividad, sobre todo en niños y más aún en niños con discapacidades, quienes tienen una forma de expresión muy diferente e incluso original, ya que con el arte terapia estaríamos fomentando una técnica innovadora y una manera diferente de trabajo con los niños, brindando atención en cada una de sus áreas, y obteniendo así la expresión y el avance en el desarrollo de cada uno de los niños. 
De la creatividad se dice que es un don que se le da a pocos, que es de ingeniosos, y al mismo tiempo, imposible de cultivar. Sin embargo, existe un método para potenciar la creatividad, de eso se encarga el arte terapia, ciencia relativamente nueva que ha dejado ver sus ventajas y eficiencia en centros educativos de Europa.

En los niños con Síndrome de Down se ha logrado sobre la discapacidad el desarrollo en las épocas de los 90 está el hecho de reconocer que las causas del retraso tienen perfiles diferentes del funcionamiento cognitivo y de lenguaje entre un niño Down y un niño aparentemente normal, es por eso que debe conocerse las diferencias y similitudes en el modo de actuar y desarrollarse de los niños con Síndrome de Down y los niños con un desarrollo normal.

Es por esto que hoy en día existen varios métodos para poder ayudar a los niños Down a desarrollar su lenguaje y poder comunicarse en su medio con las personas que desee y de una manera más profunda y poder ser entendidos, no solo por medio del lenguaje sino con muchas maneras más de comunicarse ya sea con su imaginación o corporalmente esto va en conexión con el desarrollo motor desde sus primeros meses de vida de ser un acto reflejo a ser un aprendizaje motor que va evolucionando a funciones básicas y mas complejas.

Hoy su aplicación está en el país, específicamente en Guayaquil, y por medio de este trabajo investigativo se comprobó sus beneficios sobre todo en esta población vulnerable y de la cual se sabe mucho sobre sus características genéticas, físicas, pero que se desconoce de estrategias que ayuden al desarrollo de todas sus capacidades sobre todo motoras ya que el desarrollo mental se dará por la adquisición de habilidades motoras.

\section{Conclusiones.}

- Se evaluó el desarrollo motor de 10 niños con Síndrome de Down de 5 años de edad con la Escala de Nelson Ortiz que asisten a la Fundación Corazón de María de Pelileo y se obtuvo como resultado que el $70 \%$ de los infantes tuvieron alerta en el desarrollo del área motriz gruesa, mientras que un $70 \%$ de niños se encuentra igualmente en alerta en el área motriz fina.

- Se realizó un pre-test y un post-test con la Escala de Nelson Ortiz en lo que se pudo observar en los resultados del post-test el aumento de las capacidades de los niños en sus actividades motoras.

- Se logró identificar las técnicas de Arte Terapia con los diez niños con Síndrome de Down de cinco años de edad y se obtuvo como resultado un $20 \%$ de niños en alerta en el área motriz gruesa y un $10 \%$ en el área motriz fina, como consecuencia de su aplicación durante las sesiones de terapia, de cuyos resultados se puede comprobar plenamente su efectividad logrando de esta manera un desarrollo motriz óptimo. 
- Según los datos aplicados en la t-student para observar si el Arte Terapia influye en el desarrollo motor grueso en los niños de cinco años de edad con Síndrome de Down, se pudo presenciar que si se evidencia diferencias estadísticas significativas en el desarrollo de la motricidad gruesa y en el desarrollo de la motricidad fina, concluyendo que las actividades del Arte Terapia ayudan a un mejor desarrollo motor infantil.

\section{Referencias bibliográficas.}

Martínez N. Reflexiones sobre arte terapia, arte y educación Primera ed. Arellano F, editor Madrid: Fundamentos; 2009.

Aparicio D. Las Vanguardias Artísticas en el Desarrollo del Niño. Segunda ed. Rosal, editor Chile: Publicaciones Masson; 2009.

Martínez MDL. La Intervención Arteterapéutica y su Metodología en el contexto Profesional Español. Segunda ed. Pando, editor Murcia: Departamento de Expresión Plástica, Musical y Dinámica; 2009.

López M. Creación y posibilidad aplicaciones del arte en la integración social Primera ed. Arellano, editor. Madrid: Fundamentos; 2009.

Cuervo A. Arteterapia en la Sociedad. Bravo J, editor. El Posimpresionismo del Arte terapia. Madrid: KATA; 2009. P. 13-17.

Nuñez Corral A.B. El Juego Dinámico en el Arteterapia. Segunda ed. Cao L, editor. Madrid: CADM; 2009.

Aparicio D. Las Vanguardias Artisticas en el Desarrollo del Niño. Segunda ed.

Rosal , editor. Chile : Publicaciones Masson ; 2009. (9)

Barragán JM. Arte terapia. Dinámicas entre creación y procesos terapéuticos.

Sexta ed. Aroca J, editor. Murcia: Octaedro; 2010. (1)

Cuervo A. Arteterapia en la Sociedad. Bravo J, editor. El Posimpresionismo del Arte terapia. Madrid: KATA; 2009. p. 13-17. (7) 
Garín P.B. Un Enfoque Cognitivo de las Dificultades de Aprendizaje. Segunda ed. Fernandez, editor. Madrir: Publicaciones J.A; 2010. (14)

León M. Desarrollo Motor Infantil. Segunda ed. Rubin, editor. Barcelona: Dalley T; 2009. (12)

Lopez M. Creacion y posibilidad: aplicaciones del arte en la integracion social.

Primera ed. Arellano, editor. Madrid: Fundamentos; 2009. (6)

Martinez MDL. La Intervención Arteterapéutica y su Metodología en el contexto

Profesional Español. Segunda ed. Pando, editor. Murcia:

Departamento de Expresión Plástica, Musical y Dinámica; 2009. (5)

Martínez N. Reflexiones sobre arte terapia, arte y educación. Primera ed.

Arellano F, editor. Madrid: Fundamentos ; 2009. (4)

Neisser U. Principles and Implications of Cognitive Psychology. Segunda ed.

Thomas C, editor. San Francisco : Magnolia Publishers; 2010. (10)

Nuñez Corral A.B. El Juego Dinámico en el Arteterapia. Segunda ed. Cao L, editor. Madrid : CADM; 2009. (11)

Prado F. Terapias Expresivas en la Psiquiatria. Segunda ed. Montero, editor. Barcelona: Paidós; 2010. (8)

Pueschel S.M. El Sindrome de Down y su Problemática Biomedica. Segunda ed.

E V, editor. E.E.U.U: Malchiodi C.A.; 2010. (15)

Rubin J. Creación y psicoanálisis. In Aroca J, editor. Arte terapia. Dinámicas entre creación y procesos terapéuticos. Murcia: Octaedro; 2010. p.

305. (2)

Waller D. La Formación del Arte terapia en los niños con discapacidad. Segunda ed. A G, editor. Barcelona : Publicaciones Nueva Visión ; 2010.

Linkografía. 
Rodríguez j. sitio web proyecto agrega. [online].; 2010 [cited 2015 junio 14]. Available from:

http://books.google.com.ec/books?id=rsclbqaaqbaj\&pg=pt95\&dq=el+arte+terapia.(3)

\section{CITAS BIBLIOGRÁFICAS-BASE DE DATOS UTA (MINIMO 5)}

EBRARY: Ibáñez, L. P., \& Mudarra, S. M. J. (2014). Atención temprana: diagnóstico e intervención psicopedagógica. España: UNED - Universidad Nacional de Educación a Distancia. Retrieved from http://www.ebrary.com

EBRARY: Mila, D. J. (2008). De profesión psicomotricista (2a. ed.). Argentina:

Miño y Dávila. Retrieved from http:// www. ebrary.com

EBRARY: Ovejero, H. M. (2013). Desarrollo cognitivo y motor. España: Macmillan

Iberia, S.A. Retrieved from http://site.ebrary.com/

lib/utasp/detail.action?docID=1082037439

EBRARY: La atención temprana: un compromiso con la infancia y sus familias. (2010). España: Editorial UOC. $\quad$ Retrieved from http://site.ebrary.com/lib/utasp/detail.action?docID=10609822

EBRARY: López, B. G., Alva, F. L., \& Banda, C. D. (2009). Estimulación motriz para mejorar la adquisición... de la lecto-escritura. Argentina: El Cid Editor, apuntes. Retrieved from http://site.ebrary.com/lib/utasp/ detail.action?docID=10328018 


\section{Para citar el artículo indexado.}

Troya E., Aguiar V., Peñafiel V., Miño J., Avila A \& Argotti R. (2018). El arte terapia en el desarrollo motor en los niños con síndrome de down de 5 años de edad en la fundación corazón de María de Pelileo. Revista electrónica Ciencia Digital 2(3), 513-528. Recuperado desde: http://cienciadigital.org/revistacienciadigital2/index.php/CienciaDigital/article/view/173/15 $\underline{3}$

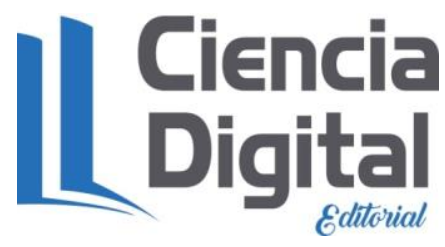

El artículo que se publica es de exclusiva responsabilidad de los autores y no necesariamente reflejan el pensamiento de la Revista Ciencia Digital.

El articulo queda en propiedad de la revista y, por tanto, su publicación parcial y/o total en otro medio tiene que ser autorizado por el director de la Revista Ciencia Digital.
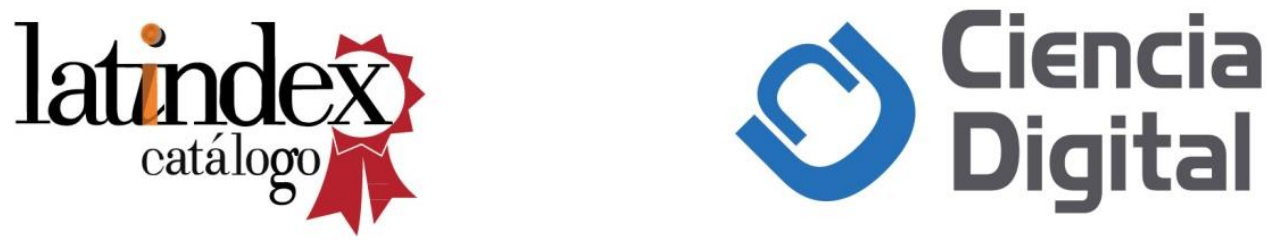